\title{
Severe Mental Illness Diagnoses and Their Association With Reoffending in a Sample of Men Adjudicated for Sexual Offences
}

\author{
Charlotte A. Aelick ${ }^{a}$, Kelly M. Babchishin ${ }^{b}$, Andrew J. R. Harris ${ }^{c}$ \\ [a] Department of Psychology, Laurentian University, Sudbury, Canada. [b] Institute of Mental Health Research, \\ University of Ottawa, Ottawa, Canada. [c] Private Practice, www.offenderrisk.com.
}

Sexual Offending: Theory, Research, and Prevention, 2020, Vol. 15(1), Article e3123, https://doi.org/10.5964/sotrap.3123

Received: 2019-10-04 • Accepted: 2020-04-10 • Published (VoR): 2020-07-22

Handling Editor: Martin Rettenberger, Centre for Criminology (Kriminologische Zentralstelle - KrimZ), Wiesbaden, Germany

Corresponding Author: Kelly M. Babchishin, University of Ottawa Institute of Mental Health Research, 1145 Carling Avenue, Ottawa, ON, K1Z 7K4, Canada. E-mail: Kelly.Babchishin@theroyal.ca

\begin{abstract}
The current study examined the relationship between mental illness and recidivism in a sample of 409 men adjudicated for sexual offences who scored higher than average on an established risk assessment tool (Static-99R). Participants were from all provincial correctional systems (except Prince Edward Island) and all regions of the Correctional Service of Canada. Severe mental illness diagnoses, with the exception of some personality disorders, were not associated with recidivism (after an average follow-up of 11 years). While some personality diagnoses were initially related to recidivism, this relationship often disappeared or was attenuated after controlling for substance misuse and risk score on the Static-99R. There were two exceptions: Histrionic and narcissistic personality disorders continued to predict sexual recidivism after controlling for Static-99R and substance misuse history. In sum, the current study suggests that severe mental illness diagnoses are not associated with higher rates of recidivism after accounting for risk score and substance misuse in men with sexual offences, with the exception of histrionic and narcissistic personality disorder diagnoses. For this reason, risk judgements that weigh both known risk factors and severe mental illness may overestimate an individual's risk to reoffend.
\end{abstract}

\section{Keywords}

severe mental illness, sex offences, recidivism, psychiatric diagnoses 


\section{Highlights}

- As severe mental illness diagnoses are more prevalent among men with sexual offences than among the general prison population; establishing a clear understanding of the role of mental illness in sexual recidivism is critical to improving intervention.

- Severe mental illnesses were not associated with higher rates of recidivism with the exception of two personality disorder diagnoses.

- Risk judgements that weigh known risk factors and severe mental illness may overestimate an individual's risk to reoffend.

- Despite the accumulating evidence that severe mental illness is not related to recidivism, symptoms associated with severe mental illness can negatively impact treatment and, as such, interventions likely need to attend to symptoms of severe mental illness, in addition to established criminogenic risk factors.

A history of mental illness diagnoses has been associated with a greater risk of criminal behaviour (Alden et al., 2007; Fazel, Sjöstedt, et al., 2010), however, it does not appear to increase the risk of recidivism among those who have previously committed an offence (Lee \& Hanson, 2016; Mann et al., 2010). Nonetheless, the tendency to equate severe mental illness with violence, unpredictability, and dangerousness is pervasive (ArboledaFlórez \& Stuart, 2012; Markowitz, 2011; Van Dorn et al., 2005). Criminalization (Mulvey \& Schubert, 2017; Skeem et al., 2014), homelessness (Arboleda-Flórez \& Stuart, 2012), social isolation (Link et al., 1999; Van Dorn et al., 2005), and unemployment (Arboleda-Flórez \& Stuart, 2012), are cited as some of the consequences associated with practical complications of having a mental illness and, in particular, the social stigmatization attached to a mental illness diagnosis. Individuals adjudicated for sexual offences diagnosed with mental illness are especially vulnerable to these perceptions given the existing stigmatization of these individuals as dangerous and socially undesirable (Tewksbury, 2005, 2012).

Studies have found that mental illness predicts the onset of offending behaviours (Bonta et al., 2014; Brennan et al., 2000; Castillo \& Fiftal Alarid, 2011; Corrigan \& Watson, 2005; Vinkers et al., 2011). The relationship between mental illness and the onset of offending, however, is often described as confounded and attributable to mental illness being associated with criminogenic risk factors for crime, such as impulsivity and substance misuse (Bonta et al., 2014; Elbogen \& Johnson, 2009; Vinkers et al., 2011). As such, it has been speculated that the relationship between mental illness and offending behaviours may be indirect (Hiday, 2006). Indeed, research suggests that factors associated with mental illnesses - such as lack of accountability (Vinkers et al., 2011), antisocial cognitions (Andrews et al., 2006), and comorbid substance use (Fazel, Lichtenstein, et al., 2010) - are more predictive of offending than mental illness diagnoses. 
Controlling for established risk factors, especially substance abuse, has been found to attenuate if not nullify the relationship between mental illness and the onset of offending (Alden et al., 2007; Brennan et al., 2000; Fazel, Lichtenstein, et al. 2010; Hiday, 2006; Matejkowski \& Ostermann, 2015; Prins et al., 2015). For example, a Danish birth cohort study found psychotic disorders comorbid with personality disorder or substance use to be strongly related to the onset of violent offending, but when controlling for demographic factors and substance use, mental illness alone was not predictive of violent offending (Brennan et al., 2000). Hiday (2006) found individuals with a major mental illness who did not abuse substances were nearly three times less likely to engage in violent behaviour than those who abuse substances both with and without a major mental disorder ( $7 \%$ vs. $18 \%$ and $22 \%$, respectively).

Other factors, such as genetics, victimization history, and socioeconomic status have also been found to confound the relationship between mental illness and the onset of offending (Fazel, Lichtenstein, et al., 2010; Hiday, 2006). A longitudinal study by Fazel, Lichtenstein, et al. (2010), for example, found individuals with a diagnosis of bipolar disorder were at a greater risk of violent crime when compared to the general population and unaffected siblings ( $O R=2.3$ and 1.6, respectively). The association between bipolar diagnosis and violent crime was found to be primarily derived from comorbid substance use $(O R=6.4)$, with limited risk of violent crime found among individuals without comorbid substance use $(O R=1.3)$. The risk of violent crime was further moderated when compared to siblings unaffected by bipolar disorder $(O R=1.1)$, suggesting genetic or shared environmental factors influence criminal behaviour rather than the presence of bipolar disorder. The finding that genetic and shared environmental factors influence the onset of criminal behaviour has also been observed in familial aggregation studies of sexual and violent crime (Frisell et al., 2011; Långström et al., 2015). When controlling for stressful life events and impaired social supports, Silver and Teasdale (2005) found the influence of mental illness to be minimized. In short, mental illness may play less of a role in predicting general and violent offending than other factors.

There are significantly fewer studies examining the role of mental illness in predicting sexual offending, however, available studies suggest similar findings to the general and violent offending literature. That is, mental illness appears to be less predictive of onset of sexual offending when controlling for established static and dynamic risk factors (Alden et al., 2007; Bonta et al., 1998). In a longitudinal study of a Danish birth cohort, Alden et al. (2007) found that individuals with a comorbid substance use disorder were significantly more likely to commit a sexual offence than those with a psychotic disorder alone. More research, however, is needed to understand the interrelationship between mental illness, substance misuse, and sexual offending.

While individuals with a history of mental illness may be at a greater risk of committing an offence than the general population (Alden et al., 2007; Fazel, Sjöstedt, et al., 2010) - likely due to its association with other known risk factors - this factor does not appear 
to increase the risk of recidivism among individuals who have previously committed a sexual offence (Lee \& Hanson, 2016; Mann et al., 2010). Mann and colleagues' (2010) found many factors associated with sexual reoffending (e.g., hostility, multiple paraphilias, offence-supportive attitudes, resistance to rules, poor problem solving, impulsivity, and negative social influences) in their review of recidivism studies - however, mental illness was not one of them.

The literature suggests that static risk factors, such as arrest history (Prins et al., 2015), and dynamic risk factors such as substance use (Alden et al., 2007; Lee \& Hanson, 2016) and other criminogenic needs (Andrews et al., 2006; Kingston et al., 2015; Peterson et al., 2014) may be more predictive of recidivism than mental illness alone. For example, studies have consistently identified substance use to be among the greatest predictors of recidivism for individuals adjudicated for sexual offences and those adjudicated for non-sexual offences (Bonta et al., 2014; Castillo \& Fiftal Alarid, 2011; Corrigan \& Watson, 2005; Kingston et al., 2015). In a large sample of individuals adjudicated for sexual offences ( $n=947)$, Lee and Hanson (2016) found that although psychiatric hospitalization was associated with an increased rate of sexual reoffending, the relationship no longer reached statistical significance after controlling for static and dynamic risk factors. Kingston and colleagues (2015) compared the effects of established risk factors using the Static-99 and a history of mental illness on sexual recidivism in a sample of 586 men with a contact sexual offence and found that established static and dynamic risk factors were most predictive of recidivism.

A meta-analysis by Bonta et al. (2014) examined the relationship between mental illness and risk of general and violent recidivism in a sample of individuals with a history of nonsexual and sexual offences. Results indicated established risk factors were significantly more predictive of recidivism than mental illness. There are some exceptions in the limited literature: In 2014, Abracen and colleagues conducted a study involving paroled offenders. This study found bipolar disorder was associated with increased rates of recidivism of any kind even after accounting for actuarial risk assessment scores (Abracen et al., 2014). Conversely, Matejkowski and Ostermann (2015) found the relationship between serious mental illness and all types of recidivism to be mediated by risk level in a random sample of individuals released on parole in the state of New Jersey. As such, previous literature seems to suggest that the variance that mental health variables have to offer is generally overtaken in the regression by more established risk factors.

Despite inconsistencies in the literature regarding the role of mental illness, some actuarial risk assessments include mental health items, which means that mental illness diagnoses increase risk ratings for these scales. Some examples include the Violence Risk Appraisal Guide (VRAG), now in its revised edition VRAG-R (Harris et al., 2015). The VRAG-R is designed to assess the likelihood of nonsexual violent or sexual contact reoffending among male offenders and Item 10 assesses indicators of a conduct disorder present before the age of 15 . While the absence of symptoms can decrease overall risk 
score by two points, a maximum symptom score can increase overall risk by five points. This, however, marks a significant change from the original VRAG which included a heavier reliance on mental illness in assessing risk of reoffending. DSM diagnoses of schizophrenia (Item 12) or any personality disorder (Item 11) were included in the VRAG and could increase the overall risk rating. While this change could indicate a greater reliance on the prevalence of conduct disorder among offenders, it may also reflect a shift in our understanding of the influence of mental illness on recidivism.

\section{The Current Study}

Establishing a clear understanding of the role of mental illness is critical to developing improved assessment tools, making evidence-based management decisions, and directing interventions for individuals adjudicated for a sexually motivated offence. The current study aims to examine the relationship between severe mental illness and recidivism in a sample of men adjudicated for a sexually motivated offence while controlling for risk factors associated with sexual offending. It was hypothesized that men adjudicated for a sexually motivated offence with a history of severe mental illness diagnoses will recidivate at higher rates than those without such diagnoses, however, that this relationship will be attenuated or nullified when controlling for established risk factors, including substance misuse history and Static-99R scores.

\section{Method}

\section{Participants}

The convenience sample included 409 men with a contact sexual offence conviction in Canada. Offenders were from all provincial correctional systems (except Prince Edward Island) and all regions of the Correctional Service of Canada. All offenders were supervised by a parole officer, probation officer, or federal police officer. ${ }^{1}$ The original data was collected in 1996 as part of a study of dynamic risk factors (see Hanson \& Harris, 1998, for full methodology). The recidivism data was later updated in 2017, providing up to a 36 year follow-up time for the current study (the average follow-up was 11 years).

A descriptive summary of the sample demographic information can be found in Table 1. A total of 16 potential mental illness diagnoses were included in the analysis. In sum, $42 \%$ of the sample had multiple diagnoses (171/409), 39\% had one diagnosis (159/409), and $19 \%$ had no diagnoses (79/409). The sample represents a group with an elevated risk to reoffend, with an average score of 4.3 on Static-99R; only 15\% of individuals adjudicated for sexual offences in Canada are expected to score higher than a score of 4 (Hanson

1) In the province of British Columbia, a small number of offenders were supervised by officers of the Royal Canadian Mounted Police. 
et al., 2012). As a result of censorship due to insufficient follow-up data or missing information on the examined variables, the final analyses included 370 individuals in the sexual and nonsexual violent forms of recidivism and 371 offenders in the sexual and any criminal offence recidivism analyses. After an average of 11.4 years follow-up (range from 0.4 to 35.6 years, $S D=8.7, M d n=9.4$ ), 25\% reoffended with a nonsexual violent offence (93/370), 32\% reoffended with a sexual offence (119/371), 44\% reoffended with a violent (including sexual) offence (164/370), and 55\% reoffended with any criminal offence (205/371). Most individuals completed some form of treatment (77\%; 285/370), which would have taken place in Canada in the late 1980's and early 1990's. At that time treatment tended to focus on the development of detailed offence cycles and offender disclosure. Risk, needs, and responsivity (RNR) sensitive treatment methodologies were not yet in common use in Canada.

\section{Table 1}

Means and Standard Deviations of Sample Demographic Information

\begin{tabular}{|c|c|c|c|c|c|c|c|c|c|c|}
\hline \multirow[b]{2}{*}{ Demographic variable } & \multicolumn{3}{|c|}{ Complete sample } & \multicolumn{3}{|c|}{$\begin{array}{c}\text { No Severe } \\
\text { Mental Illness }\end{array}$} & \multicolumn{3}{|c|}{$\begin{array}{c}\text { Any Severe } \\
\text { Mental Illness }\end{array}$} & \multirow{2}{*}{$\begin{array}{l}\text { Cohen's } d \\
{[95 \% \mathrm{CI}]}\end{array}$} \\
\hline & $M$ & $S D$ & $N$ & $\boldsymbol{M}$ & $S D$ & $N$ & $M$ & $S D$ & $\boldsymbol{N}$ & \\
\hline Age at index offence & 34.95 & 10.91 & 407 & 36.67 & 12.43 & 95 & 34.43 & 10.37 & 312 & $0.21[-0.02,0.44]$ \\
\hline Static-99R total score & 4.30 & 2.39 & 407 & 3.89 & 2.31 & 95 & 4.44 & 2.40 & 312 & $\mathbf{0 . 2 3}[0.001,0.46]$ \\
\hline Any substance misuse history ${ }^{\mathrm{a}}$ & $58 \%$ & 235 & 409 & $50 \%$ & 48 & 97 & $60 \%$ & 187 & 312 & $0.26[-0.04,0.50]$ \\
\hline \# of prior sexual offences & 2.33 & 3.96 & 409 & 2.09 & 3.13 & 97 & 2.41 & 4.18 & 312 & $0.08[-0.15,0.30]$ \\
\hline \# of prior nonsexual violent offences & 1.25 & 2.46 & 409 & 0.95 & 2.32 & 97 & 1.35 & 2.50 & 312 & $0.16[-0.07,0.39]$ \\
\hline \# of prior offences & 7.13 & 12.97 & 409 & 5.01 & 8.66 & 97 & 7.79 & 13.99 & 312 & $0.22[-0.01,0.44]$ \\
\hline
\end{tabular}

Note. Cohen's $d$ comparing those with any severe mental illness diagnoses with those without any severe mental illness diagnoses. Positive $d s$ indicate that individuals with severe mental illness had more risk-relevant factors than those without severe mental illness. Age was reverse scored so that a positive $d$ indicates that those with severe mental illness were younger than those without severe mental illness. Bolded values represent a statistically significant group difference $p<.05$.

${ }^{a}$ Any substance misuse is reported as \%, number with substance misuse, and total sample size.

\section{Measures}

\section{Demographic}

As part of the original data collection, a standardized coding manual was developed to record background information from each offender's file and national criminal history record. The background information included various static factors such as offence history, substance misuse, and basic identifying information.

\section{Severe Mental IIIness Diagnoses}

The presence in the person's community correctional file of severe mental illness diagnoses any time before file coding occurred, was recorded in the coding manual. These diag- 
noses were made by clinicians when the individual was assessed in the mental health, correctional, and in some cases, the educational system. The diagnoses were, in some cases, diagnosed after the index offence, however, the clinician was examining if mental illness occurred prior to the offence. For the majority of the sample, the diagnoses would have been made at their first contact with the mental health or correctional systems and in some cases the educational system. In the 1990's some risk assessment methodologies included mental illness items, most prominently, the Risk Assessment Guide (RAG; Harris et al., 1993; Webster et al., 1994).

Severe mental illness included: mood disorder (i.e., bipolar, depression), personality disorder (i.e., antisocial, avoidant, borderline, dependent, histrionic, narcissistic, obsessive compulsive, paranoid, schizoid, and schizotypal personality disorders), attention deficit hyperactivity disorder (ADHD), anxiety, conduct disorder, and traumatic brain injury. Appendix A provides the diagnostic criteria for histrionic, narcissistic, and schizotypal personality disorders from the Diagnostic and Statistical Manual of Mental Disorders (American Psychiatric Association, 2013).

\section{Substance Misuse}

Community supervision coded for presence of alcohol abuse and illicit drug use anytime throughout the supervision period. For the purpose of this study, we combined alcohol and illicit drug use into a substance misuse variable.

\section{Static-99R}

Static-99R (Hanson \& Thornton, 2000; Helmus et al., 2012) is an empirically derived actuarial risk assessment tool designed to predict sexual recidivism in adult male persons convicted of sexually motivated offences (also see www.static99.org). It has 10 items used to create a total score ranging from -3 to 12 . The Static-99R items are identical to those of Static-99 with the exception of updated age weights. In the current dataset, we computed Static-99R score from syntax as we had Static-99 scores (i.e., requires recomputing the age weight).

\section{Recidivism}

The current study included four measures of recidivism defined as any charges or convictions for new crimes: (a) any crime recidivism including sexual, violent, and general offences, excluding breaches of conditional orders; (b) sexual recidivism including any offence deemed to be sexual in nature, which includes both contact and noncontact sexual offences; (c) violent (including sexual) recidivism includes any offence causing harm to another person (i.e., contact and nonsexual violence); (d) nonsexual violent recidivism includes any violent offence, excluding those deemed to be sexual in nature. Recidivism information was collected from official information from the Canadian Police Information Centre (CPIC) in 2017. 


\section{Data Analyses}

Cox regression analyses, which allow for controlling of variable follow-up time, were used to assess the relationship between mental illness and recidivism rates. Hazard ratios $(\operatorname{Exp}[\mathrm{B}])$ indicate the degree to which recidivism increases or decreases based on mental illness diagnosis. If the $95 \%$ confidence interval for the hazard ratio includes 0 , then the association is not statistically significant $(p>.05)$. For all forms of recidivism, the mental health diagnosis was entered on the first block, while Static-99R and substance misuse were added simultaneously on the second block. This allowed for investigation of the effect of the Static-99R score and substance misuse on any significant relationship between mental illness and recidivism. Subsequent analyses included interactions between mental illness diagnosis and substance use as well as mental illness diagnosis and Static-99R score if the combination models reached statistical significance. These analyses yielded no significant interaction effects. A summary of these results can be found in Appendix B. Cohen's $d$ for continuous variables were computed as

$$
d=\frac{M_{1}-M_{2}}{S_{w}}
$$

where the pooled standard deviation was

$$
S_{w}=\sqrt{\frac{\left(N_{1}-1\right)\left(S D_{1}\right)^{2}+\left(N_{2}-1\right)\left(S D_{2}\right)^{2}}{N_{1}-1+N_{2}-1}}
$$

and the variance

$$
\frac{N_{1}+N_{2}}{N_{1} N_{2}}+\frac{d^{2}}{2\left(N_{1}+N_{2}\right)}
$$

(Cohen, 1988). Cohen's $d$ was estimated from dichotomous variables using the formula from Sánchez-Meca et al. (2003) and adding .5 to each cell to allow for $d$ calculation with empty cells (Fleiss, 1994). Specifically, the $d$ was estimated as

$$
d=\frac{1}{1.65}\left[\ln \left(\frac{\{a+.5\}\{d+.5\}}{\{b+.5\}\{c+.5\}}\right)\right]
$$

and its variance as

$$
0.3673\left(\frac{1}{a+.5}+\frac{1}{b+.5}+\frac{1}{c+.5}+\frac{1}{d+.5}\right)
$$




\section{Results}

More often than not, severe mental illness was not predictive of sexual recidivism among this sample of higher risk individuals. Personality disorders (specifically, histrionic and narcissistic personality disorders) were bivariately related to sexual recidivism (see Table 2). After controlling for substance misuse and Static-99R scores, both histrionic and narcissistic personality disorders continued to predict sexual recidivism, although the relationships were reduced.

\section{Table 2}

Hazard Rate of Diagnoses, Substance Misuse, and Static-99R With Sexual Recidivism $\left(n_{\text {recidivists }}=119 / 371\right)$

\begin{tabular}{|c|c|c|c|c|}
\hline \multirow[b]{3}{*}{ Diagnoses ( $n$ diagnosed/Total $N$ ) } & \multicolumn{4}{|c|}{ Hazard Ratio [95\% CI] } \\
\hline & \multirow[t]{2}{*}{ Unadjusted model } & \multicolumn{3}{|c|}{ Adjusted model } \\
\hline & & Diagnosis & $\begin{array}{c}\text { Substance } \\
\text { misuse }\end{array}$ & Static-99R \\
\hline $\operatorname{ADHD}(7 / 371)$ & $1.20[0.30,4.86]$ & $0.84[0.21,3.39]$ & $1.12[0.77,1.63]$ & $1.35[1.24,1.47]$ \\
\hline Any mood disorder $(78 / 371)$ & $0.77[0.47,1.26]$ & $0.67[0.41,1.10]$ & $1.13[0.78,1.64]$ & $1.36[1.25,1.48]$ \\
\hline Bipolar (6/371) & $0.48[0.07,3.47]$ & $0.24[0.03,1.74]$ & $1.12[0.78,1.63]$ & $1.37[1.25,1.49]$ \\
\hline Depression $(73 / 371)$ & $0.79[0.48,1.31]$ & $0.75[0.45,1.24]$ & $1.35[0.78,1.63]$ & $1.35[1.24,1.48]$ \\
\hline Any personality disorder $(294 / 371)$ & $1.73[1.11,2.71]$ & $1.48[0.93,2.33]$ & $1.05[0.73,1.54]$ & $1.34[1.23,1.46]$ \\
\hline Antisocial personality $(277 / 372)$ & $1.32[0.88,1.98]$ & $1.25[0.83,1.87]$ & $1.24[0.87,1.77]$ & $1.28[1.18,1.39]$ \\
\hline Avoidant (11/371) & $0.25[0.04,1.77]$ & $0.25[0.04,1.78]$ & $1.10[0.76,1.59]$ & $1.35[1.24,1.47]$ \\
\hline Borderline (16/371) & $1.13[0.42,3.06]$ & $0.95[0.35,2.58]$ & $1.12[0.77,1.63]$ & $1.35[1.24,1.47]$ \\
\hline Dependent $(39 / 371)$ & $0.73[0.37,1.45]$ & $0.87[0.44,1.73]$ & $1.13[0.78,1.63]$ & $1.35[1.24,1.47]$ \\
\hline Histrionic $(9 / 371)$ & $4.27[1.86,9.82]$ & $3.33[1.45,7.68]$ & $1.23[0.78,1.63]$ & $1.34[1.23,1.47]$ \\
\hline Obsessive compulsive (16/371) & $1.05[0.43,2.57]$ & $1.05[0.43,2.58]$ & $1.12[0.77,1.62]$ & $1.35[1.24,1.47]$ \\
\hline Paranoid (8/371) & $1.35[0.43,4.24]$ & $1.27[0.40,4.02]$ & $1.23[0.78,1.64]$ & $1.35[1.24,1.47]$ \\
\hline Narcissistic (56/371) & $2.13[1.37,3.31]$ & $1.68[1.07,2.63]$ & $1.09[0.75,1.58]$ & $1.34[1.23,1.46]$ \\
\hline Schizoid $(20 / 371)$ & $1.27[0.56,2.90]$ & $1.20[0.53,2.74]$ & $1.12[0.89,1.11]$ & $1.35[1.24,1.47]$ \\
\hline Schizotypal $(7 / 371)^{\mathrm{a}}$ & $0.05[-.0001,16.55]$ & & & \\
\hline Anxiety (7/371) & $0.47[0.07,3.38]$ & $0.33[0.05,2.34]$ & $1.11[0.77,1.61]$ & $1.36[1.25,1.48]$ \\
\hline Conduct disorder ${ }^{\mathrm{b}}(234 / 370)$ & $1.40[0.97,2.02]$ & $1.20[0.83,1.74]$ & $1.22[0.86,1.75]$ & $1.28[1.18,1.38]$ \\
\hline Traumatic brain injury $(2 / 371)$ & $2.51[0.35,17.95]$ & $1.66[0.23,11.95]$ & $1.11[0.77,1.62]$ & $1.35[1.24,1.47]$ \\
\hline
\end{tabular}

Note. The unadjusted model provides the bivariate relationship between severe mental illness diagnoses and recidivism. The adjusted model provides the relationship that is adjusted for substance misuse and Static-99R scores. Bolded values are statistically significant at $p<.05$.

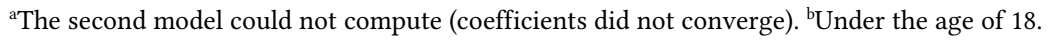

The computed variable 'any personality disorder diagnosis' and narcissistic personality disorder were also bivariately associated with violent recidivism (see Table 3), although the hazard ratios were lower than for sexual recidivism. After controlling for substance misuse and Static-99R score, these diagnoses were no longer associated with recidivism. 


\section{Table 3}

Hazard Rate of Diagnoses, Substance Misuse, and Static-99R With Violent (Including Sexual) Recidivism ( $n_{\text {recidivists }}=$ 164/370)

\begin{tabular}{|c|c|c|c|c|}
\hline \multirow[b]{3}{*}{ Diagnoses $(n$ diagnosed/Total $N$ ) } & \multicolumn{4}{|c|}{ Hazard ratio $[95 \% \mathrm{CI}]$} \\
\hline & \multirow[t]{2}{*}{ Unadjusted model } & \multicolumn{3}{|c|}{ Adjusted model } \\
\hline & & Diagnosis & $\begin{array}{c}\text { Substance } \\
\text { misuse }\end{array}$ & Static-99R \\
\hline $\operatorname{ADHD}(7 / 370)$ & $0.78[0.19,3.14]$ & $0.50[0.12,2.02]$ & $1.63[1.18,2.26]$ & $1.35[1.25,1.45]$ \\
\hline Any mood disorder $(78 / 370)$ & $0.83[0.55,1.25]$ & $0.71[0.47,1.07]$ & $1.62[1.18,2.27]$ & $1.64[1.25,1.45]$ \\
\hline Bipolar (6/370) & $1.34[0.43,4.21]$ & $0.60[0.19,1.92]$ & $1.63[1.18,2.26]$ & $1.35[1.25,1.45]$ \\
\hline Depression $(73 / 370)$ & $0.77[0.51,1.18]$ & $0.72[0.47,1.10]$ & $1.63[1.18,2.26]$ & $1.35[1.25,1.45]$ \\
\hline Any personality disorder $(294 / 370)$ & $1.63[1.12,2.36]$ & $1.32[0.90,1.93]$ & $1.56[1.12,2.17]$ & $1.34[1.24,1.44]$ \\
\hline Antisocial personality $(277 / 370)$ & $1.18[0.82,1.70]$ & $1.08[0.75,1.55]$ & $1.61[1.16,2.23]$ & $1.34[1.25,1.44]$ \\
\hline Avoidant (11/370) & $0.58[0.18,1.80]$ & $0.59[0.19,1.86]$ & $1.60[1.16,2.22]$ & $1.35[1.25,1.45]$ \\
\hline Borderline $(16 / 370)$ & $0.19[0.53,2.72]$ & $0.96[0.43,2.18]$ & $1.62[1.17,2.24]$ & $1.34[1.24,1.44]$ \\
\hline Dependent $(39 / 370)$ & $0.78[0.44,1.37]$ & $0.89[0.50,1.58]$ & $1.62[1.17,2.25]$ & $1.35[1.44,2.25]$ \\
\hline Histrionic $(9 / 370)$ & $1.75[0.72,4.28]$ & $1.35[0.55,2.03]$ & $1.62[1.17,2.25]$ & $1.34[1.25,1.44]$ \\
\hline Narcissistic (56/370) & $1.85[1.24,2.75]$ & $1.36[0.91,3.30]$ & $1.60[1.15,2.21]$ & $1.34[1.24,1.44]$ \\
\hline Obsessive compulsive $(16 / 370)$ & $1.05[0.49,2.23]$ & $1.09[0.51,2.32]$ & $1.62[1.17,2.24]$ & $1.35[1.25,1.44]$ \\
\hline Paranoid (8/370) & $1.01[0.32,3.17]$ & $1.05[0.33,3.32]$ & $1.62[1.17,2.25]$ & $1.34[1.25,1.44]$ \\
\hline Schizoid $(20 / 370)$ & $1.08[0.53,2.20]$ & $0.92[0.45,1.88]$ & $1.62[1.17,2.24]$ & $1.34[1.25,1.44]$ \\
\hline Schizotypal $(7 / 370)$ & $1.31[0.42,4.12]$ & $1.52[0.48,4.76]$ & $1.62[1.17,2.24]$ & $1.34[1.25,1.44]$ \\
\hline Anxiety $(7 / 370)$ & $0.30[0.04,2.17]$ & $0.21[0.03,1.51]$ & $1.61[1.16,2.23]$ & $1.35[1.25,1.45]$ \\
\hline Conduct disorder $^{\mathrm{a}}(234 / 370)$ & $1.28[0.93,1.78]$ & $1.07[0.76,1.48]$ & $1.60[1.16,2.23]$ & $1.34[1.25,1.44]$ \\
\hline Traumatic brain injury $(2 / 370)$ & $1.66[0.23,11.86]$ & $0.93[0.13,6.64]$ & $1.62[1.17,2.24]$ & $1.34[1.25,1.45]$ \\
\hline
\end{tabular}

Note. The unadjusted model provides the bivariate relationship between severe mental illness diagnoses and recidivism. The adjusted model provides the relationship that is adjusted for substance misuse and Static-99R scores. Bolded values are statistically significant at $p<.05$.

${ }^{\mathrm{a}}$ Under the age of 18.

We also examined the relationship between diagnoses and any crime and nonsexual violent offending and had similar findings (see Table 4 and Table 5, respectively). Schizotypal personality disorder continued to be predictive of nonsexual violence after controlling for substance misuse and Static-99R. Noteworthy is that both antisocial personality disorder and conduct disorder in childhood were not predictive of recidivism in the current sample. 
Table 4

Hazard Rate of Diagnoses, Substance Misuse and Static-99R With Any Crime $\left(n_{\text {recidivists }}=205 / 371\right)$

\begin{tabular}{|c|c|c|c|c|}
\hline \multirow[b]{3}{*}{ Diagnoses ( $n$ diagnosed/Total $N$ ) } & \multicolumn{4}{|c|}{ Hazard ratio $[95 \% \mathrm{CI}]$} \\
\hline & \multirow{2}{*}{$\begin{array}{c}\text { Unadjusted model } \\
\text { Diagnosis }\end{array}$} & \multicolumn{3}{|c|}{ Adjusted model } \\
\hline & & Diagnosis & $\begin{array}{c}\text { Substance } \\
\text { misuse }\end{array}$ & Static-99R \\
\hline $\operatorname{ADHD}(7 / 371)$ & $0.55[0.14,2.19]$ & $0.33[0.08,1.33]$ & $1.68[1.25,2.24]$ & $1.33[1.24,1.42]$ \\
\hline Any mood disorder $(78 / 371)$ & $1.06[0.75,1.50]$ & $0.93[0.66,1.31]$ & $1.66[1.24,2.22]$ & $1.33[1.24,1.42]$ \\
\hline Bipolar (6/371) & $1.55[0.58,4.18]$ & $0.99[0.37,2.66]$ & $1.65[1.24,2.21]$ & $1.33[1.24,1.42]$ \\
\hline Depression $(73 / 371)$ & $0.99[0.69,1.41]$ & $0.88[0.62,1.26]$ & $1.66[1.24,2.22]$ & $1.33[1.24,1.42]$ \\
\hline Any personality disorder $(294 / 371)$ & $1.51[1.09,2.08]$ & $1.21[0.87,1.69]$ & $1.61[1.20,2.16]$ & $1.32[1.23,1.41]$ \\
\hline Antisocial personality $(277 / 371)$ & $1.07[0.78,1.46]$ & $0.95[0.69,1.30]$ & $1.66[1.24,2.22]$ & $1.33[1.24,1.42]$ \\
\hline Avoidant (11/371) & $0.40[0.13,1.26]$ & $0.40[0.13,1.25]$ & $1.64[1.22,2.19]$ & $1.33[1.24,1.42]$ \\
\hline Borderline $(16 / 371)$ & $0.84[0.38,1.90]$ & $0.65[0.29,1.47]$ & $1.67[1.25,2.24]$ & $1.33[1.24,1.42]$ \\
\hline Dependent $(39 / 371)$ & $0.76[0.46,1.27]$ & $0.84[0.50,1.40]$ & $1.67[1.24,2.23]$ & $1.32[1.24,1.41]$ \\
\hline Histrionic $(9 / 371)$ & $1.63[0.72,3.69]$ & $1.20[0.53,2.71]$ & $1.65[1.24,2.21]$ & $1.32[1.24,1.42]$ \\
\hline Narcissistic $(56 / 371)$ & $1.65[1.14,2.37]$ & $1.17[0.81,1.70]$ & $1.63[1.22,2.19]$ & $1.32[1.24,1.41]$ \\
\hline Obsessive compulsive $(16 / 371)$ & $0.90[0.44,1.82]$ & $0.84[0.42,1.72]$ & $1.66[1.24,2.22]$ & $1.33[1.24,1.42]$ \\
\hline Paranoid (8/371) & $1.12[0.41,3.00]$ & $1.13[0.42,3.05]$ & $1.66[1.24,2.22]$ & $1.33[1.24,1.42]$ \\
\hline Schizoid (20/371) & $0.89[0.45,1.71]$ & $0.75[0.39,1.47]$ & $1.66[1.24,2.22]$ & $1.33[1.24,1.42]$ \\
\hline Schizotypal $(7 / 371)$ & $1.55[0.58,4.17]$ & $1.93[0.71,5.21]$ & $1.64[1.23,2.20]$ & $1.33[1.24,1.42]$ \\
\hline Anxiety $(7 / 371)$ & $0.48[0.12,1.95]$ & $0.33[0.08,1.32]$ & $1.64[1.23,2.20]$ & $1.33[1.25,1.42]$ \\
\hline Conduct disorder $^{\mathrm{a}}(234 / 371)$ & $1.15[0.86,1.53]$ & $0.91[0.68,1.22]$ & $1.68[1.25,2.26]$ & $1.33[1.24,1.42]$ \\
\hline Traumatic brain injury $(2 / 371)$ & $1.18[0.17,8.39]$ & $0.63[0.09,4.50]$ & $1.66[1.24,2.22]$ & $1.33[1.24,1.42]$ \\
\hline
\end{tabular}

Note. The unadjusted model provides the bivariate relationship between severe mental illness diagnoses and recidivism. The adjusted model provides the relationship that is adjusted for substance misuse and Static-99R scores. Bolded values are statistically significant at $p<.05$.

${ }^{\mathrm{a}}$ Under the age of 18.

The base rates of these diagnoses were quite high (63\%-74\%), regardless of recidivism status. For example, 78\% (105/135) of sexual recidivists and 73\% (172/236) of sexual nonrecidivists had an antisocial personality diagnosis and 69\% (93/135) of sexual recidivists and $60 \%(141 / 236)$ of sexual nonrecidivists had a conduct disorder diagnosis. ${ }^{2}$

2) Stemming from a reviewer's comment, additional exploratory analyses was conducted to investigate the influence of multiple diagnoses (any diagnoses) on recidivism ( $0=$ no diagnosis; $1=$ one diagnosis; $2=$ two or more diagnoses; significant results are marked with an *). Any recidivism: $0=0.68,95 \% \mathrm{CI}=[0.47,0.99]^{*} ; 1=1.11,95 \% \mathrm{CI}=$ $[0.84,1.47] ; 2=1.15,95 \% \mathrm{CI}=[0.87,1.52]$; sexual recidivism: $0=0.53,95 \% \mathrm{CI}=[0.31,0.91]^{*} ; 1=1.19,95 \% \mathrm{CI}=[0.83$, $1.72] ; 2=1.21,95 \% \mathrm{CI}=[0.84,1.73]$; nonsexual violent recidivism: $0=0.58,95 \% \mathrm{CI}=[0.31,1.05] ; 1=1.11[0.73,1.67] ; 2$ $=1.24[0.82,1.86]$; violent (including sexual) recidivism: $0=0.60,95 \% \mathrm{CI}=[0.39,0.93]^{*} ; 1=1.15,95 \% \mathrm{CI}=[0.84,1.56]$; $2=1.18,95 \% \mathrm{CI}=[0.87,1.61]$. 


\section{Table 5}

Hazard Rate of Diagnoses, Substance Misuse and Static-99R With Non-Sexual Violent Recidivism $\left(n_{\text {recidivists }}=\right.$ 93/370)

\begin{tabular}{|c|c|c|c|c|}
\hline \multirow[b]{3}{*}{ Diagnoses $(n$ diagnosed/Total $N$ ) } & \multicolumn{4}{|c|}{ Hazard ratio $[95 \% \mathrm{CI}]$} \\
\hline & \multirow[t]{2}{*}{ Unadjusted model } & \multicolumn{3}{|c|}{ Adjusted model } \\
\hline & & Diagnosis & $\begin{array}{l}\text { Substance } \\
\text { misuse }\end{array}$ & Static-99R \\
\hline ADHD $(7 / 370)$ & $0.72[0.10,5.17]$ & $0.46[0.06,3.31]$ & $2.48[1.54,4.01]$ & $1.31[1.19,1.45]$ \\
\hline Any mood disorder $(78 / 370)$ & $0.94[0.56,1.60]$ & $0.84[0.49,1.42]$ & $2.47[1.53,3.99]$ & $1.32[1.19,1.45]$ \\
\hline Bipolar (6/370) & $1.75[0.43,7.11]$ & $0.86[0.21,3.54]$ & $2.47[1.53,3.99]$ & $1.31[1.19,1.45]$ \\
\hline Depression $(73 / 370)$ & $0.86[0.50,1.50]$ & $0.81[0.47,1.41]$ & $2.47[1.53,3.99]$ & $1.31[1.19,1.45]$ \\
\hline Any personality disorder $(294 / 370)$ & $1.86[1.10,3.14]$ & $1.37[0.80,2.35]$ & $2.36[1.46,3.83]$ & $1.30[1.18,1.43]$ \\
\hline Antisocial personality $(277 / 370)$ & $1.15[0.70,1.86]$ & $0.98[0.60,1.60]$ & $2.47[1.53,4.00]$ & $1.31[1.19,1.45]$ \\
\hline Avoidant (11/370) & $0.70[0.17,2.85]$ & $0.86[0.21,3.52]$ & $2.46[1.52,3.97]$ & $1.31[1.19,1.45]$ \\
\hline Borderline (16/370) & $2.24[0.98,5.12]$ & $1.82[0.79,4.18]$ & $2.44[1.51,3.94]$ & $1.31[1.19,1.44]$ \\
\hline Dependent $(39 / 370)$ & $0.72[0.33,1.56]$ & $0.83[0.38,1.79]$ & $2.47[1.53,3.99]$ & $1.31[1.19,1.44]$ \\
\hline Histrionic $(9 / 370)$ & $1.02[0.25,4.14]$ & $0.80[0.20,3.24]$ & $2.46[1.52,3.97]$ & $1.31[1.19,1.45]$ \\
\hline Narcissistic (56/370) & $1.71[1.02,2.87]$ & $1.19[0.70,2.00]$ & $2.45[1.52,3.96]$ & $1.30[1.18,1.44]$ \\
\hline Obsessive compulsive $(16 / 370)$ & $1.34[0.54,3.30]$ & $1.41[0.57,3.47]$ & $2.46[1.53,3.97]$ & $1.31[1.19,1.44]$ \\
\hline Paranoid (8/370) & $1.33[0.33,5.39]$ & $1.67[0.41,6.83]$ & $2.50[1.55,4.05]$ & $1.31[1.19,1.45]$ \\
\hline Schizoid (20/370) & $1.17[0.48,2.89]$ & $0.98[0.40,2.42]$ & $2.47[1.53,3.98]$ & $1.31[1.19,1.45]$ \\
\hline Schizotypal (7/370) & $2.66[0.84,8.41]$ & $3.23[1.02,10.28]$ & $2.47[1.53,3.99]$ & $1.31[1.19,1.45]$ \\
\hline Anxiety $(7 / 370)$ & $0.59[0.08,4.26]$ & $0.48[0.07,3.45]$ & $2.45[1.52,3.96]$ & $1.31[1.19,1.45]$ \\
\hline Conduct disorder ${ }^{\mathrm{a}}(234 / 370)$ & $1.12[0.73,1.73]$ & $0.87[0.56,1.35]$ & $2.52[1.55,4.08]$ & $1.32[1.19,1.45]$ \\
\hline Traumatic brain injury $(2 / 370)^{\mathrm{b}}$ & $0.50\left[5.10 \mathrm{E}^{-7},-4761.37\right]$ & - & - & - \\
\hline
\end{tabular}

Note. Bolded values are statistically significant at $p<.05$.

${ }^{a}$ Under the age of 18 . ${ }^{b}$ In part due to low base rate (2 of 370), the second model could not compute (coefficients did not converge).

\section{Discussion}

The relationship between severe mental illness and reoffending among men with a history of sexually motivated offences is unclear. Individuals with a history of mental illness may be at greater risk of committing an offence than the general population (Alden et al., 2007; Fazel, Sjöstedt, et al., 2010) - yet this factor does not appear to increase the risk of recidivism among men who have previously committed a sexual offence (Lee \& Hanson, 2016; Mann et al., 2010). Some studies, however, have found an association between mental illness diagnoses and all forms of recidivism resulting in revocation or suspension of conditional release (Abracen et al., 2014; Matejkowski \& Ostermann, 2015). The current study aimed to elucidate the relationship between serious mental illness (diagnosed prior to the index sexual offence) and long-term recidivism in a sample of 409 men adjudicated for a sexually motivated offence in Canada with the prevalence of different diagnoses in this sample ranging from $0.5 \%-72 \%$ (median $3 \%$ ). In 
most cases, severe mental illness was not predictive of any form of recidivism similar to a meta-analysis of individuals convicted of sexual and nonsexual offences (Bonta et al., 2014). While some personality diagnoses were initially related to recidivism, this relationship often disappeared or was attenuated after controlling for substance misuse and risk score on the Static-99R. These findings are consistent with studies that found established risk factors to be more predictive of recidivism than mental illness alone in sample of individuals convicted of sexual and nonsexual offences (Alden et al., 2007; Bonta et al., 2014; Castillo \& Fiftal Alarid, 2011; Lee \& Hanson, 2016).

\section{Practical Implications}

Our study suggests mental illness may play an indirect role in recidivism. Within the scope of an indirect model, individuals may not have offence-related symptoms but the presence of a mental illness may contribute to reoffending by influencing criminogenic needs (Lee \& Hanson, 2016). Specifically, the bivariate relationship between severe mental illness and recidivism seems to be an indirect relationship attributed to severe mental illness being a predictor of known risk factors for recidivism in general (Haig, 2003). For this reason, risk judgements that weigh both known risk factors and severe mental illness may overestimate an individual's risk to reoffend. Longitudinal studies would be useful to examine the extent to which changes in severe mental illness influences risk factors.

Certain exceptions, however, may apply in the event that symptoms of mental illness are related to offending behaviours. In the current study, histrionic and narcissistic personality disorders continued to predict sexual recidivism even when substance misuse and risk rating were considered. Histrionic personality disorder is defined as a pattern of attention seeking behaviours, dramatization, and excessive emotionality. In comparison, narcissistic personality disorder is characterized by an inflated sense of self-importance, a lack of empathy for others, and a need for attention or admiration. Although the relationship between histrionic and narcissistic personality disorders and recidivism diminished after controlling for known risk factors, these diagnoses continued to reach statistical significance thresholds for sexual recidivism, suggesting a relationship between these forms of personality disorder and sexual recidivism. This finding concurs with previous studies that found some personality disorders to be associated with various forms of recidivism (Abracen et al., 2014; Eher et al., 2010; Howard et al., 2008).

In contrast to the current study, Abracen and colleagues (2014) found borderline personality disorder to be predictive of recidivism in a sample of paroled offenders. Similarly, Howard and colleagues (2008) found both antisocial and borderline personality disorders to be associated with the onset of offending in a sample of 224 (109 male and 115 female) adults who met the criteria for a diagnosis of at least one personality disorder according to the DSM-IV. It is important to note, however, that the sample for Howard et al. (2008) included volunteers in a clinical trial, did not require any forensic history as a prerequisite for participation, and did not assess recidivism. These previous 
studies posited that factors associated with the aforementioned personality disorders may facilitate offending behaviours (Abracen et al., 2014; Howard et al., 2008).

Antisocial personality disorder is identified as a pervasive pattern of recklessness, impulsivity, irresponsibility, and violation of the rights of others. Similarly, borderline personality disorder is characterized by impulsivity, instability in relationships, and difficulty regulating emotions. Given that sexual offences are considered interpersonal violations, symptoms of personality disorders may influence an individual's interpersonal style and likelihood of committing a sexual offence (Looman et al., 2005). For this reason, differences in the criteria used to classify offence history and recidivism between these studies and the current study may account for differences in the personality disorders found to be associated with increased risk of recidivism. Limited research has examined the relationship between these variables (Abracen et al., 2014). While most forms of severe mental illness did not increase risk of recidivism of any kind after accounting for risk rating and substance misuse in this study, personality disorders appear to have some link to recidivism (Abracen et al., 2014; Eher et al., 2010; Howard et al., 2008). Future research should investigate the relationship between personality disorders and recidivism, especially using an unselected sample of individuals convicted of sexually motivated offences and accounting for participation in treatment during follow-up times. Our sample was mostly treated (77\%), though we did not have information on the content of the treatment program and anticipate that mental illness symptoms would not necessarily have been targeted.

Future studies examining the relationship between symptoms of mental illnesses, rather than the diagnosis, would be essential in further clarifying the link between severe mental illness and offending behaviours, for both the onset of offending and recidivism. Symptoms common to personality disorders identified in the current study (histrionic and narcissistic personality disorders) and previous studies (borderline and antisocial personality disorders), such as impulsivity, shallow affect, hostility, and problems with self-regulation, have been linked to offending behaviours (Howard et al., 2008; Mann et al., 2010). These factors are related to increased risk of recidivism among men with sexually motivated offences (Mann et al., 2010) and may be present independent of a mental disorder. Therefore, it is likely that these characteristics independently increase risk of recidivism.

Mental illness is thought to negatively impact treatment outcome and may influence an individual's ability to receive treatment (Wong \& Olver, 2015). Often these conditions are associated with denial, minimization, aggression, manipulative behaviours, and high rates of attrition in treatment (Abracen \& Looman, 2004; Looman et al., 2005; Wong \& Olver, 2015). Psychiatric symptoms have also been found to interfere with treatment engagement and cooperation with supervision (Craissati \& Beech, 2001; Stinson, 2016; Völlm et al., 2019). As such, despite the accumulating evidence that mental illness is not 
associated with recidivism, their associated symptoms is considered a responsivity ${ }^{3}$ issue in treatment (Wong \& Olver, 2015).

Taken as a whole, addressing both criminogenic needs and the symptoms of mental illness are likely required to effectively reduce recidivism (Barnao et al., 2010; Eher et al., 2019). Attending to symptoms of mental illness is especially relevant to treatment programs designed specifically for men with sexually motivated offences. A cross-sectional study involving 1,346 individuals convicted of a sexual offence found significantly higher prevalence of at least one mental illness diagnosis (92.1\%) compared to both general prison populations $(48 \%-73 \%)$ and the general population $(20 \%-30 \%$ lifetime prevalence; Eher et al., 2019). Studies investigating the association of symptoms of mental illness with recidivism would inform the development of improved intervention programs. Assessment procedures prior to treatment may screen for these symptoms, in order to develop effective intervention plans. By taking into account both the criminogenic needs and responsivity issues associated with symptoms of serious mental illnesses, more effective intervention plans can be established.

\section{Limitations}

In defining mental illness, the current study included only those offenders who had a formal diagnosis prior to or at intake for the index sexual offence. As a result, those who may have exhibited symptoms of severe mental illness, but who had no diagnosis, were not included. In addition, changes in diagnoses and symptoms were not assessed. In order to examine the validity of an indirect influence of mental illness on recidivism, it would be important to examine the extent to which changes in symptoms of severe mental illness is associated with changes in risk factors. This study also did not differentiate between type of sex offences committed when examining the influence of mental illness on recidivism. The current sample included individuals adjudicated for sexually motivated offences against both adults and children; however, these distinctions were not included in the analyses. This study also did not differentiate between type of sexual offences committed when examining the influence of mental illness on recidivism. Future studies could consider this factor when examining the link between mental illness and sexual recidivism. Furthermore, we were unable to account for treatments used to address symptoms of mental illness and it is quite possible that pharmaceutical (i.e., antipsychotic medications) or therapy-based interventions could have a role in further mediating the influence of mental health on recidivism.

The sample is unique and high risk. The sample score at the $80^{\text {th }}$ percentile on their risk to reoffend compared to other men adjudicated for a sexually motivated offence in Canada, as assessed by the Static-99R, having an average score of 4.3 . Only $15 \%$ of

3) Responsivity is the skill of delivering interventions in a manner that takes into account the individual's learning style, current abilities, and current difficulties (Andrews \& Bonta, 2010). 
men adjudicated for a sexual offence in Canada are expected to score higher than a score of 4 of the Static-99R. There are several factors that contributed to this sample's high risk status. The sample originated from a study that was designed with a $50 \%$ prior recidivism rate, (408 recidivists / 401 non-recidivists; Hanson \& Harris, 1998) and the presence of prior sexual offences is one of the strongest predictors of sexual recidivism (Hanson \& Bussière, 1996). We then updated the dataset to include a longer recidivism period (11.4 years on average but up to 35 years for some offenders in the sample). A follow-up length of this nature would have caught most recidivists (e.g., Hanson et al., 2018). Perhaps less obviously, the sample of origin involved an extended interview with the supervising officer who were often keen to discuss cases they saw as complex, interesting, and difficult. As a result, it is possible that more high risk or precarious cases were sampled.

Even with a sample of close to 400 participants, two models could not converge, which is symptomatic of issues regarding base rate and insufficient variability in scores of the predictors. We did not increase the maximum amount of iteration to force a finding and instead left these two analyses as missing as increased iterations would have produced unreliable results. Larger sample sizes, with a larger variability in Static-99R scores, along with an unbiased base rate of substance misuse and diagnoses, could improve these models. For example, it is a robust finding in criminology that conduct disorder and antisocial personality disorders predict recidivism (e.g., Mann et al., 2010) but the current study did not find this relationship reached statistical significance. The rate of these disorders, however, was high $(63 \%-74 \%)$. It is likely that the lack of variability on Static-99R, substance misuse history, and these personality disorders precluded statistical significance (Cox, 1972).

\section{Conclusion}

Severe mental illness diagnoses, as a general rule, are not related to recidivism among men adjudicated for sexually motivated offences. The current study found that only some psychiatric (mostly personality disorder) diagnoses were bivariately associated with recidivism, and that these relationships were reduced after controlling for established risk factors as assessed by the Static-99R and substance misuse history. As such, we conclude that the observed relationship between severe mental illness and recidivism is likely indirect and explained by the association between mental illness diagnoses and established risk factors. For this reason, risk judgements that weigh both known risk factors and severe mental illness may overestimate an individual's risk to reoffend. Personality diagnoses (and specifically histrionic and narcissistic personality disorders) were the exception, being predictive of recidivism even after controlling for substance misuse and Static-99R scores. We hypothesize that the predictive ability of these personality diagnoses is due to their common symptomology (e.g., impulsivity, shallow affect, hostility, and problems with self-regulation), which have been found to be predictive of 
recidivism (e.g., Mann et al., 2010). Finally, symptoms associated with certain forms of severe mental illness may negatively impact treatment and, as such, symptoms of severe mental illness should be considered a responsivity issue in treatment.

Funding: The authors have no funding to report.

Competing Interests: The authors have declared that no competing interests exist.

Acknowledgments: The authors have no support to report.

Data Availability: The data used for the present study is the property of the Government of Canada. Therefore, we are not able to make it publicly available. The anonymized data could be released but would require a data sharing agreement with the Government of Canada. We (the authors) are not copyright holders of the material. However, interested researchers could always contact the corresponding author to start the process.

\section{References}

Abracen, J., Langton, C. M., Looman, J., Gallo, A., Ferguson, M., Axford, M., \& Dickey, R. (2014). Mental health diagnoses and recidivism in paroled offenders. International fournal of Offender Therapy and Comparative Criminology, 58, 765-779. https://doi.org/10.1177/0306624X13485930

Abracen, J., \& Looman, J. (2004). Issues in the treatment of sexual offenders: Recent developments and directions for future research. Aggression and Violent Behavior, 9, 229-246.

https://doi.org/10.1016/S1359-1789(01)00074-X

Alden, A., Brennan, P., Hodgins, S., \& Mednick, S. (2007). Psychotic disorders and sex offending in a Danish birth cohort. Archives of General Psychiatry, 64, 1251-1258. https://doi.org/10.1001/archpsyc.64.11.1251

American Psychiatric Association. (2013). Diagnostic and statistical manual of mental disorders: DSM-5 (5th ed.). Washington, DC, USA: Author.

Andrews, D. A., \& Bonta, J. (2010). The psychology of criminal conduct (5th ed.). Cincinnati, OH, USA: Anderson Publishing.

Andrews, D. A., Bonta, J., \& Wormith, J. S. (2006). The recent past and near future of risk and/or need assessment. Crime and Delinquency, 52, 7-27. https://doi.org/10.1177/0011128705281756

Arboleda-Flórez, J., \& Stuart, H. (2012). From sin to science: Fighting the stigmatization of mental illnesses. Canadian fournal of Psychiatry, 57, 457-463.

https://doi.org/10.1177/070674371205700803

Barnao, M., Robertson, P., \& Ward, T. (2010). Good lives model applied to a forensic population. Psychiatry, Psychology and Law, 17, 202-217. https://doi.org/10.1080/13218710903421274

Bonta, J., Blais, J., \& Wilson, H. A. (2014). A theoretically informed meta-analysis of the risk for general and violent recidivism for mentally disordered offenders. Aggression and Violent Behavior, 19, 278-287. https://doi.org/10.1016/j.avb.2014.04.014 
Bonta, J., Law, M., \& Hanson, R. K. (1998). The prediction of criminal and violent recidivism among mentally disordered offenders: A meta-analysis. Psychological Bulletin, 123, 123-142. https://doi.org/10.1037/0033-2909.123.2.123

Brennan, P. A., Mednick, S. A., \& Hodgins, S. (2000). Major mental disorders and criminal violence in a Danish birth cohort. Archives of General Psychiatry, 57, 494-500. https://doi.org/10.1001/archpsyc.57.5.494

Castillo, E. D., \& Fiftal Alarid, L. (2011). Factors associated with recidivism among offenders with mental illness. International fournal of Offender Therapy and Comparative Criminology, 55, 98-117. https://doi.org/10.1177/0306624X09359502

Cohen, J. (1988). Statistical power analysis for the behavioral sciences (2nd ed.). Hillsdale, NJ, USA: Lawrence Erlbaum.

Corrigan, P. W., \& Watson, A. C. (2005). Mental illness and dangerousness: Fact or misperception, and implications for stigma. In P. W. Corrigan (Ed.), On the stigma of mental illness: Practical strategies for research and social change (pp. 165-179). Washington, DC, USA: American Psychological Association. https://doi.org/10.1037/10887-007

Cox, D. R. (1972). Regression models and life-tables. Journal of the Royal Statistical Society: Series B. Methodological, 34, 187-202. https://doi.org/10.1111/j.2517-6161.1972.tb00899.x

Craissati, J., \& Beech, A. (2001). Attrition in a community treatment program for child sexual abusers. Journal of Interpersonal Violence, 16, 205-221. https://doi.org/10.1177/088626001016003002

Eher, R., Rettenberger, M., Matthes, A., \& Schilling, F. (2010). Stable dynamic risk factors in child sexual abusers: The incremental predictive power of narcissistic personality traits beyond the Static-99/Stable-2007 priority categories on sexual reoffense. Sexual Offender Treatment, 5, 1-12. Retrieved from http://www.sexual-offender-treatment.org/index.php?id=83\&type=123

Eher, R., Rettenberger, M., \& Turner, D. (2019). The prevalence of mental disorders in incarcerated contact sexual offenders. Acta Psychiatrica Scandinavica, 139, 572-581. https://doi.org/10.1111/acps.13024

Elbogen, E. B., \& Johnson, S. C. (2009). The intricate link between violence and mental disorder: Results from the National Epidemiologic Survey on Alcohol and Related Conditions. Archives of General Psychiatry, 66, 152-161. https://doi.org/10.1001/archgenpsychiatry.2008.537

Fazel, S., Lichtenstein, P., Grann, M., Goodwin, G. M., \& Långström, N. (2010). Bipolar disorder and violent crime: New evidence from population-based longitudinal studies and systematic review. Archives of General Psychiatry, 67, 931-938. https://doi.org/10.1001/archgenpsychiatry.2010.97

Fazel, S., Sjöstedt, G., Grann, M., \& Långström, N. (2010). Sexual offending in women and psychiatric disorder: A national case-control study. Archives of Sexual Behavior, 39, 161-167. https://doi.org/10.1007/s10508-008-9375-4

Fleiss, J. L. (1994). Measures of effect size for categorical data. In H. Cooper \& L. V. Hedges (Eds.), The handbook of research synthesis (pp. 245-260). New York, NY, USA: Russell Sage Foundation. 
Frisell, T., Lichtenstein, P., \& Langstrom, N. (2011). Violent crime runs in families: A total population study of 12.5 million individuals. Psychological Medicine, 41, 97-105. https://doi.org/10.1017/S0033291710000462

Haig, B. D. (2003). What is a spurious correlation? Understanding Statistics, 2, 125-132. https://doi.org/10.1207/S15328031US0202_03

Hanson, R. K., \& Bussière, M. T. (1996). Predictors of sexual offender recidivism: A meta-analysis (User Report 96-04). Ottawa, Canada: Department of the Solicitor General of Canada.

Hanson, R. K., \& Harris, A. J. R. (1998). Dynamic predictors of sexual recidivism. Ottawa, Canada: Solicitor General Canada.

Hanson, R. K., Harris, A. J. R., Letourneau, E., Helmus, L. M., \& Thornton, D. (2018). Reductions in risk based on time offense-free in the community: Once a sexual offender, not always a sexual offender. Psychology, Public Policy, and Law, 24, 48-63. https://doi.org/10.1037/law0000135

Hanson, R. K., Lloyd, C. D., Helmus, L. M., \& Thornton, D. (2012). Developing non-arbitrary metrics for risk communication: Percentile ranks for the Static-99R and Static-2002R sexual offender risk tools. International fournal of Forensic Mental Health, 11, 9-23. https://doi.org/10.1080/14999013.2012.667511

Harris, G. T., Rice, M. E., \& Quinsey, V. L. (1993). Violent recidivism of mentally disordered offenders: The development of a statistical prediction instrument. Criminal fustice and Behavior, 20, 315-335. https://doi.org/10.1177/0093854893020004001

Harris, G. T., Rice, M. E., Quinsey, V. L., \& Cormier, C. A. (2015). Violent offenders: Appraising and managing risk (3rd ed.). Washington, DC, USA: American Psychological Association.

Hanson, R. K., \& Thornton, D. (2000). Improving risk assessments for sex offenders: A comparison of three actuarial scales. Law and Human Behavior, 24, 119-136.

https://doi.org/10.1023/A:1005482921333

Helmus, L., Thornton, D., Hanson, R. K., \& Babchishin, K. M. (2012). Improving the predictive accuracy of Static-99 and Static-2002 with older sex offenders: Revised age weights. Sexual Abuse: A fournal of Research and Treatment, 24, 64-101.

https://doi.org/10.1177/1079063211409951

Hiday, V. A. (2006). Putting community risk in perspective: A look at correlations, causes and controls. International fournal of Law and Psychiatry, 29, 316-331.

https://doi.org/10.1016/j.ijlp.2004.08.010

Howard, R. C., Huband, N., Duggan, C., \& Mannion, A. (2008). Exploring the link between personality disorder and criminality in a community sample. Fournal of Personality Disorders, 22, 589-603. https://doi.org/10.1521/pedi.2008.22.6.589

Kingston, D. A., Olver, M. E., Harris, M., Wong, S. C., \& Bradford, J. M. (2015). The relationship between mental disorder and recidivism in sexual offenders. International fournal of Forensic Mental Health, 14, 10-22. https://doi.org/10.1080/14999013.2014.974088

Långström, N., Babchishin, K. M., Fazel, S., Lichtenstein, P., \& Frisell, T. (2015). Sexual offending runs in families: A 37-year nationwide study. International fournal of Epidemiology, 44, 713-720. https://doi.org/10.1093/ije/dyv029 
Lee, S. C., \& Hanson, R. K. (2016). Recidivism risk factors are correlated with a history of psychiatric hospitalization among sex offenders. Psychological Services, 13, 261-271. https://doi.org/10.1037/ser0000081

Link, B. G., Phelan, J. C., Bresnahan, M., Stueve, A., \& Pescosolido, B. A. (1999). Public conceptions of mental illness: Labels, causes, dangerousness, and social distance. American fournal of Public Health, 89, 1328-1333. https://doi.org/10.2105/AJPH.89.9.1328

Looman, J., Dickie, I., \& Abracen, J. (2005). Responsivity issues in the treatment of sexual offenders. Trauma, Violence \& Abuse, 6, 330-353. https://doi.org/10.1177/1524838005280857

Mann, R. E., Hanson, R. K., \& Thornton, D. (2010). Assessing risk for sexual recidivism: Some proposals on the nature of psychologically meaningful risk factors. Sexual Abuse, 22, 191-217. https://doi.org/10.1177/1079063210366039

Markowitz, F. E. (2011). Mental illness, crime, and violence: Risk, context, and social control. Aggression and Violent Behavior, 16, 36-44. https://doi.org/10.1016/j.avb.2010.10.003

Matejkowski, J., \& Ostermann, M. (2015). Serious mental illness, criminal risk, parole supervision, and recidivism: Testing of conditional effects. Law and Human Behavior, 39, 75-86. https://doi.org/10.1037/lhb0000094

Mulvey, E. P., \& Schubert, C. A. (2017). Mentally ill individuals in jails and prisons. Crime and fustice, 46, 231-277. https://doi.org/10.1086/688461

Peterson, J. K., Skeem, J., Kennealy, P., Bray, B., \& Zvonkovic, A. (2014). How often and how consistently do symptoms directly precede criminal behavior among offenders with mental illness? Law and Human Behavior, 38, 439-449. https://doi.org/10.1037/lhb0000075

Prins, S. J., Skeem, J. L., Mauro, C., \& Link, B. G. (2015). Criminogenic factors, psychotic symptoms, and incident arrests among people with serious mental illnesses under intensive outpatient treatment. Law and Human Behavior, 39, 177-188. https://doi.org/10.1037/lhb0000104

Sánchez-Meca, J., Chacón-Moscoso, S., \& Marín-Martínez, F. (2003). Effect-size indices for dichotomized outcomes in meta-analysis. Psychological Methods, 8, 448-467. https://doi.org/10.1037/1082-989X.8.4.448

Silver, E., \& Teasdale, B. (2005). Mental disorder and violence: An examination of stressful life events and impaired social support. Social Problems, 52, 62-78.

https://doi.org/10.1525/sp.2005.52.1.62

Skeem, J. L., Winter, E., Kennealy, P. J., Louden, J. E., Tatar, I. I., \& Joseph, R. (2014). Offenders with mental illness have criminogenic needs too: Toward recidivism reduction. Law and Human Behavior, 38, 212-224. https://doi.org/10.1037/lhb0000054

Stinson, J. D. (2016). Predictors of treatment noncompletion in a sample of inpatient sex offenders with serious mental illness. Psychiatric Services, 67, 43-48. https://doi.org/10.1176/appi.ps.201400415

Tewksbury, R. (2005). Collateral consequences of sex offender registration. Journal of Contemporary Criminal fustice, 21, 67-81. https://doi.org/10.1177/1043986204271704

Tewksbury, R. (2012). Stigmatization of sex offenders. Deviant Behavior, 33, 606-623. https://doi.org/10.1080/01639625.2011.636690 
Van Dorn, R. A., Swanson, J. W., Elbogen, E. B., \& Swartz, M. S. (2005). A comparison of stigmatizing attitudes toward persons with schizophrenia in four stakeholder groups: Perceived likelihood of violence and desire for social distance. Psychiatry, 68, 152-163. https://doi.org/10.1521/psyc.2005.68.2.152

Vinkers, D. J., De Beurs, E., Barendregt, M., Rinne, T., \& Hoek, H. W. (2011). The relationship between mental disorders and different types of crime. Criminal Behaviour and Mental Health, 21, 307-320. https://doi.org/10.1002/cbm.819

Völlm, B., Craissati, J., Grubin, D., \& Skett, S. (2019). Learning from research: Adapting interventions for sexual offending to improve outcomes. Criminal Behaviour and Mental Health, 29, 227-238. https://doi.org/10.1002/cbm.2122

Webster, C. D., Harris, G. T., Rice, C., \& Quinsey, V. L. (1994). The violence prediction scheme: Assessing dangerousness in high risk men. Toronto, Canada: Centre of Criminology, University of Toronto.

Wong, S. C., \& Olver, M. E. (2015). Risk reduction treatment of psychopathy and applications to mentally disordered offenders. CNS Spectrums, 20, 303-310.

https://doi.org/10.1017/S1092852915000322

\section{Appendices}

\section{Appendix A: Diagnostic Criteria From the Diagnostic and Statistical Manual of Mental Disorders Version 5 (DSM-5)}

\section{Histrionic Personality Disorder}

A pervasive pattern of excessive emotionality and attention seeking, beginning by early adulthood and present in a variety of contexts, as indicated by five (or more) of the following:

1. Is uncomfortable in situations in which he or she is not the center of attention.

2. Interaction with others is often characterized by inappropriate sexually seductive or provocative behavior.

3. Displays rapidly shifting and shallow expression of emotions.

4. Consistently uses physical appearance to draw attention to self.

5. Has a style of speech that is excessively impressionistic and lacking in detail.

6. Shows self-dramatization, theatricality, and exaggerated expression of emotion.

7. Is suggestible (i.e., easily influenced by others or circumstances).

8. Considers relationships to be more intimate than they actually are. (DSM5; 301.50 [F60.4])

\section{Narcissistic Personality Disorder}

A pervasive pattern of grandiosity (in fantasy or behavior), need for admiration, and lack of empathy, beginning by early adulthood and present in a variety of contexts, as indicated by five (or more) of the following:

1. Has a grandiose sense of self-importance (e.g., exaggerates achievements and talents, expects to be recognized as superior without commensurate achievements). 
2. Is preoccupied with fantasies of unlimited success, power, brilliance, beauty, or ideal love.

3. Believes that he or she is "special" and unique and can only be understood by, or should associate with, other special or high-status people (or institutions).

4. Requires excessive admiration.

5. Has a sense of entitlement (i.e., unreasonable expectations of especially favorable treatment or automatic compliance with his or her expectations).

6. Is interpersonally exploitative (i.e., takes advantage of others to achieve his or her own ends).

7. Lacks empathy: is unwilling to recognize or identify with the feelings and needs of others.

8. Is often envious of others or believes that others are envious of him or her. Shows arrogant, haughty behaviors or attitudes. (DSM-5, 301.81 [F60.81])

\section{Schizotypal Personality Disorder}

A pervasive pattern of social and interpersonal deficits marked by acute discomfort with, and reduced capacity for, close relationships as well as by cognitive or perceptual distortions and eccentricities of behavior, beginning by early adulthood and present in a variety of contexts, as indicated by five (or more) of the following:

1. Ideas of reference (excluding delusions of reference).

2. Odd beliefs or magical thinking that influences behavior and is inconsistent with subcultural norms (e.g., superstitiousness, belief in clairvoyance, telepathy, or "sixth sense"; in children and adolescents, bizarre fantasies or preoccupations).

3. Unusual perceptual experiences, including bodily illusions.

4. Odd thinking and speech (e.g., vague, circumstantial, metaphorical, overelaborate, or stereotyped).

5. Suspiciousness or paranoid ideation.

6. Inappropriate or constricted affect.

7. Behavior or appearance that is odd, eccentric, or peculiar.

8. Lack of close friends or confidants other than first-degree relatives.

9. Excessive social anxiety that does not diminish with familiarity and tends to be associated with paranoid fears rather than negative judgments about self.

Does not occur exclusively during the course of schizophrenia, a bipolar disorder or depressive disorder with psychotic features, another psychotic disorder, or autism spectrum disorder.

Note: If criteria are met prior to the onset of schizophrenia, add "premorbid," e.g., "schizotypal personality disorder (premorbid)." (DSM-5; 301.22 [F21]) 


\section{Appendix B: Supplemental Analyses}

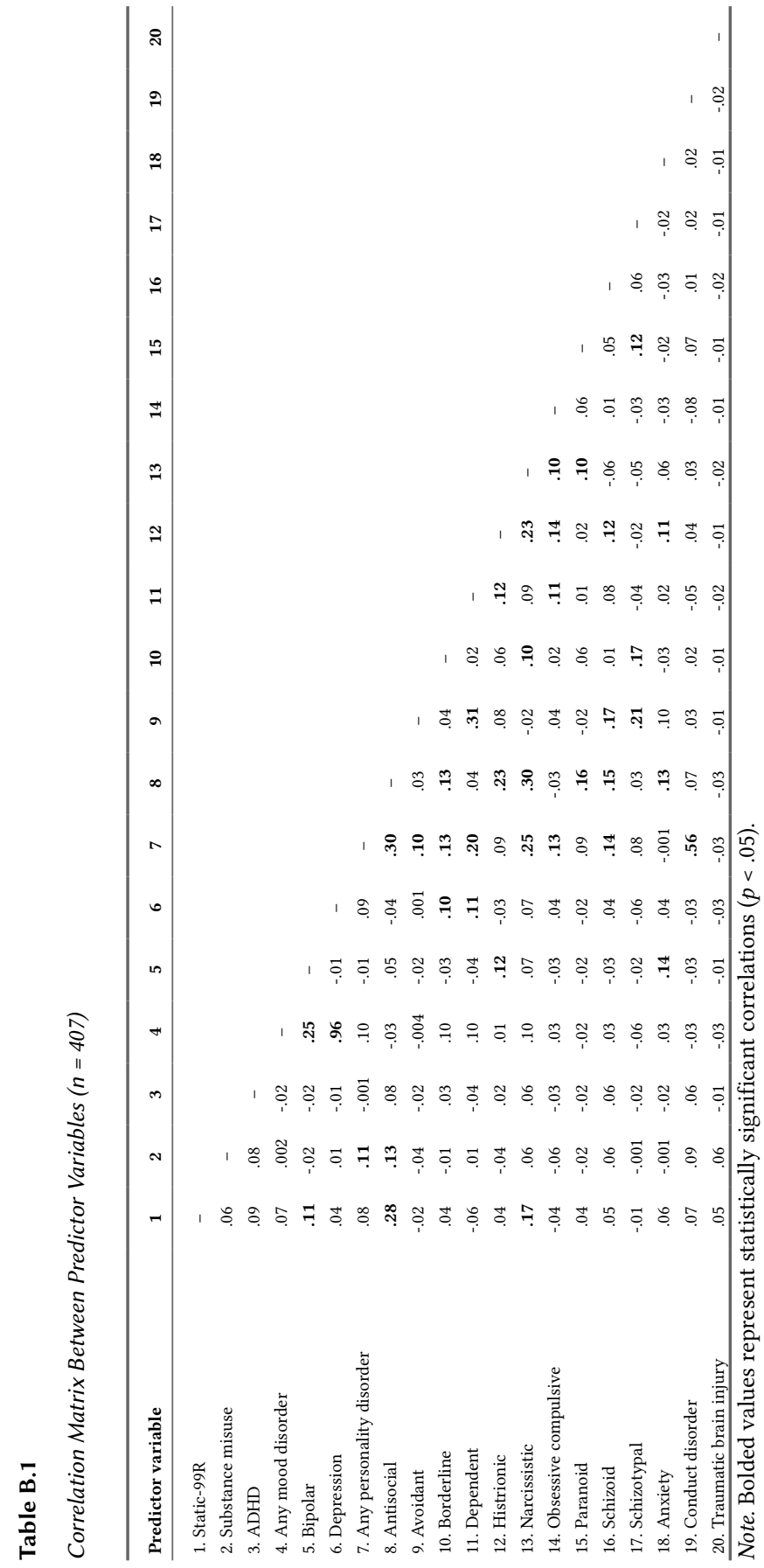




\section{Table B.2}

Summary of Recidivism Types with Substance Use and Static-99 Interaction

\begin{tabular}{|c|c|c|c|c|c|}
\hline \multirow{3}{*}{$\begin{array}{l}\text { Diagnoses }(n \\
\text { diagnosed / Total } N)\end{array}$} & \multicolumn{5}{|c|}{ Hazard ratio (95\% CI) } \\
\hline & \multirow{2}{*}{$\frac{\text { Model } 1}{\text { Diagnoses }}$} & \multicolumn{4}{|c|}{ Model 2} \\
\hline & & Diagnoses & Substance Misuse & Static-99R & Diagnosis*Substance \\
\hline \multicolumn{6}{|c|}{ Sexual recidivism with substance misuse interaction $\left(n_{\text {recidivists }}=119 / N=371\right)$} \\
\hline Histrionic (9/371) & $0.23[0.10,0.54]$ & $0.17[0.06,0.49]$ & $0.84[0.57,1.23]$ & $1.35[1.24,1.47]$ & $0.31[0.06,1.80]$ \\
\hline Narcissistic (56/371) & $2.13[1.37,3.31]$ & $2.10[1.01,4.38]$ & $0.86[0.57,1.30]$ & $1.34[1.23,1.46]$ & $0.71[0.29,1.79]$ \\
\hline \multicolumn{6}{|c|}{ Sexual recidivism with Static-99R interaction $\left(n_{\text {recidivists }}=119 / N=371\right)$} \\
\hline Histrionic (9/371) & $0.23[0.10,0.54]$ & $0.16[0.01,2.38]$ & $0.89[0.61,1.28]$ & $1.35[1.24,1.47]$ & $0.90[0.59,1.37]$ \\
\hline Narcissistic (56/371) & $2.13[1.37,3.31]$ & $0.53[0.09,3.05]$ & $0.91[0.63,1.32]$ & $1.31[1.19,1.44]$ & $1.21[0.92,1.58]$ \\
\hline \multicolumn{6}{|c|}{ Violent recidivism with substance misuse interaction $\left(n_{\text {recidivists }}=164 / N=370\right.$ ) } \\
\hline Histrionic $(9 / 370)$ & $0.57[0.23,1.39]$ & $0.47[0.15,1.50]$ & $0.60[0.43,0.84]$ & $1.34[1.25,1.44]$ & $0.41[0.07,2.52]$ \\
\hline Narcissistic $(56 / 370)$ & $1.85[1.24,2.75]$ & $1.51[0.74,3.11]$ & $0.61[0.43,0.87]$ & $1.33[1.24,1.44]$ & $0.86[0.36,2.03]$ \\
\hline \multicolumn{6}{|c|}{ Violent recidivism with Static-99R interaction $\left(n_{\text {recidivists }}=164 / N=370\right)$} \\
\hline Histrionic $(9 / 370)$ & $0.57[0.23,1.39]$ & $0.36[0.01,10.47]$ & $0.62[0.44,0.85]$ & $1.34[1.25,1.47]$ & $0.90[0.54,1.51]$ \\
\hline Narcissistic (56/370) & $1.85[1.24,2.75]$ & $1.16[0.28,4.74]$ & $0.63[0.45,0.87]$ & $1.33[1.23,1.44]$ & $1.03[0.82,1.29]$ \\
\hline \multicolumn{6}{|c|}{ Any crime with substance misuse interaction $\left(n_{\text {recidivists }}=205 / N=371\right)$} \\
\hline Histrionic $(9 / 371)$ & $0.61[0.27,1.38]$ & $0.35[0.13,0.97]$ & $0.57[0.43,0.77]$ & $1.33[1.24,1.42]$ & $0.19[0.03,1.09]$ \\
\hline Narcissistic (56/371) & $1.65[1.14,2.37]$ & $1.67[0.87,3.20]$ & $0.57[0.41,0.78]$ & $1.32[1.24,1.41]$ & $0.61[0.28,1.33]$ \\
\hline \multicolumn{6}{|c|}{ Any crime with Static-99R interaction $\left(n_{\text {recidivists }}=205 / N=371\right)$} \\
\hline Histrionic (9/371) & $0.61[0.27,1.38]$ & $0.43[0.03,6.39]$ & $0.60[0.45,0.81]$ & $1.33[1.24,1.42]$ & $0.90[0.59,1.37]$ \\
\hline Narcissistic (56/371) & $1.65[1.14,2.37]$ & $0.93[0.26,3.30]$ & $0.61[0.46,0.82]$ & $1.31[1.22,1.41]$ & $1.04[0.85,1.27]$ \\
\hline \multicolumn{6}{|c|}{ Nonsexual violence with substance misuse interaction $\left(n_{\text {recidivists }}=93 / N=370\right)$} \\
\hline Histrionic $(9 / 370)$ & $0.98[0.24,4.00]$ & $0.72[0.10,5.38]$ & $0.39[0.24,0.65]$ & $1.31[1.19,1.44]$ & $0.40[0.02,6.74]$ \\
\hline Narcissistic $(56 / 370)$ & $1.71[1.02,2.87]$ & $1.26[0.42,3.75]$ & $0.40[0.24,0.68]$ & $1.30[1.18,1.44]$ & $0.90[0.27,3.18]$ \\
\hline \multicolumn{6}{|c|}{ Nonsexual recidivism with Static-99R interaction $\left(n_{\text {recidivists }}=93 / N=370\right)$} \\
\hline Histrionic $(9 / 370)$ & $0.98[0.24,4.00]$ & $1.89^{\mathrm{E}+16}\left[0.00,2.35^{\mathrm{E}+191}\right]$ & $0.41[0.25,0.66]$ & $1.31[1.19,1.44]$ & $215.55\left[1.52^{\mathrm{E}-159}, 2.23^{\mathrm{E}+27}\right]$ \\
\hline Narcissistic $(56 / 370)$ & $1.71[1.02,2.87]$ & $2.51[0.45,14.09]$ & $0.41[0.25,0.66]$ & $1.33[1.19,1.48]$ & $0.88[0.67,1.17]$ \\
\hline
\end{tabular}

Note.. Bolded values are statistically significant at $\mathrm{p}<.05$.

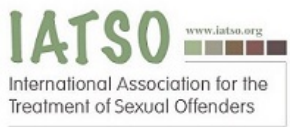

Sexual Offending: Theory, Research, and Prevention is the official journal of the International Association for the Treatment of Sexual Offenders (IATSO).

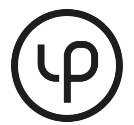

leibniz-psychology.org

PsychOpen GOLD is a publishing service by Leibniz Institute for Psychology Information (ZPID), Germany. 